\title{
General-purpose computer program for shape optimization of engineering structures using the boundary element
} method

DOI:

10.1016/0045-7949(95)00002-X

\section{Document Version}

Accepted author manuscript

Link to publication record in Manchester Research Explorer

Citation for published version (APA):

Tafreshi, A., \& Fenner, R. T. (1995). General-purpose computer program for shape optimization of engineering structures using the boundary element method. Computers and Structures, 56(5), 713-720.

https://doi.org/10.1016/0045-7949(95)00002-X

\section{Published in:}

Computers and Structures

\section{Citing this paper}

Please note that where the full-text provided on Manchester Research Explorer is the Author Accepted Manuscript or Proof version this may differ from the final Published version. If citing, it is advised that you check and use the publisher's definitive version.

\section{General rights}

Copyright and moral rights for the publications made accessible in the Research Explorer are retained by the authors and/or other copyright owners and it is a condition of accessing publications that users recognise and abide by the legal requirements associated with these rights.

\section{Takedown policy}

If you believe that this document breaches copyright please refer to the University of Manchester's Takedown Procedures [http://man.ac.uk/04Y6Bo] or contact uml.scholarlycommunications@manchester.ac.uk providing relevant details, so we can investigate your claim.

\section{OPEN ACCESS}




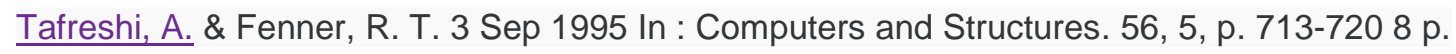

\title{
GENERAL-PURPOSE COMPUTER PROGRAM FOR SHAPE OPTIMIZATION OF ENGINEERING STRUCTURES USING THE BOUNDARY ELEMENT METHOD
}

\author{
A TAFRESHI Mechanical Engineering Division, The School of Engineering, \\ University of Manchester, Manchester, UK \\ R T FENNER Department of Mechanical Engineering, Imperial College of Science, \\ Technology and Medicine, London, UK
}

\begin{abstract}
Optimization of the shape of structural components is often concerned with minimizing stress concentration effects to ensure a longer fatigue life. A general purpose computer program, STRESOPT, for shape optimal design of two-dimensional structures in order to smooth stress peaks is presented. The program has three main parts : the stress analyser, design sensitivity analyzer and optimizer. The stress analyzer and design sensitivity analyzer use the boundary element method with isoparametric quadratic boundary elements. The boundary element method is very suitable for shape optimization and in comparison with the finite element method needs much less data, related only to the boundary of the structure being considered. Because a differentiated form of the boundary integral equation (on which the boundary element is based) can be used directly to determine the derivatives of the objective and constraint functions, the accuracy of computation is very high. The numerical optimization method used in the program is the extended penalty function approach, using the BFGS variable metric for unconstrained minimization together with Golden Section method for the one-dimensional search. Initial mesh preparation and regeneration of the boundary elements during the iterative process of optimization is both straightforward and fast. Hermitian cubic splines are well suited for the boundary shape representation and complex geometries can be described in a very compact way by a small number of design variables. Applications of the program to the optimum shape design of fillets and holes of inappropriate shape in plates and bars are presented.
\end{abstract}

\section{INTRODUCTION}

Shape optimization is an important area of current development in mechanical and structural design. Computerized procedures using mathematical programming techniques can iteratively determine the optimum shape of a component while satisfying some objectives, without at the 
same time violating the design constraints. There are various choices for the objective function. For example, minimum weight can be selected as the objective function, subject to constraints upon stresses, displacements and natural frequencies.

During the optimization process the shape of the object is continuously changing and it is important to maintain a good representation of this shape in order to preserve the accuracy of the stress analysis and the sensitivity analysis for calculating derivatives of objective and constraint functions with respect to the design variables. Geometric design variables are chosen which characterize either the shape of an exterior boundary or the shape and location of an interior cutout. Stress analysis techniques such as the boundary element or the finite element method can be coupled to the numerical optimization techniques [1-4].

This paper describes the implementation of numerical techniques in a general-purpose computer program, "STRESOPT" to perform shape optimization in two-dimensions for smoothing stress peaks under geometric constraints [5]. The main parts of this FORTRAN program carry out numerical optimization, stress analysis, sensitivity analysis, shape representation and automatic mesh generation. In the sensitivity analysis, the first partial derivatives of structural response with respect to design variables provide the link between mathematical optimization techniques and stress analysis methods in order to reach the optimum in a reasonable number of iterations. The boundary element method which, being a boundary-oriented method is particularly suitable for shape optimization is used for elastostatic stress analysis. It offers significant advantages over the finite element method, including a reduced set of equations, simple data preparation to run a problem, and good resolution of stresses. A directly differentiated form of the boundary integral equation with respect to geometric design variables is used to calculate shape design sensitivities [5-7]. Hermitian cubic spline functions used to represent boundary shapes offer considerable advantages in fitting a wide range of geometries, and in the automatic remeshing process. A computer aided design package IDEAS (Integrated Design Engineering Analysis Software) [8] is used as a pre- and post-processor.

Practical applications of the program are concerned with optimum profile design of fillets and holes in plates and bars. 


\section{NUMERICAL OPTIMIZATION METHOD}

In designing engineering structures and components, various factors have to be taken into consideration. In particular, localized high stresses measured by stress concentration factors relative to appropriate mean stresses, should be avoided. One way of smoothing out local stress peaks is to minimize the square of the deviation of the stresses from a desired uniform average stress, which would be related to the maximum permissible stress in the material. In this case, the objective function can be written as

$$
F(X)=\int\left(\sigma_{e}-\sigma_{a}\right)^{2} d s
$$

where the $X_{i}$ are geometric design variables (coordinates of points on the movable part of the boundary), $\sigma_{e}$ is the local von-Mises equivalent stress, $\sigma_{a}$ is the desired average stress, and $\mathrm{s}$ is the distance measured along the boundary. Note that only stresses on the boundary are sampled. This is for two reasons: boundary element analysis yields boundary stresses more readily and economically than interior values, and in the vast majority of practical problems the greatest stresses in a component occur at its surface.

The optimization method used here is the extended penalty function [1], and for the unconstrained minimization the BFGS variable metric method [9-11] is employed. These methods are very suitable because the objective function is highly nonlinear, while the geometrical constraints are linear. The approach is to minimize the objective function as an unconstrained function but to provide some penalty to limit constraint violations. This requires the solution of several unconstrained minimization problems in obtaining the optimum constrained design.

The unconstrained method is treated iteratively, and the form of iteration is given by

$$
X^{q}=X^{q-1}+\alpha_{q} S^{q}
$$

where $\mathrm{q}$ is the iteration number, $\mathrm{X}$ is the vector of design variables, $\mathrm{S}^{\mathrm{q}}$ is the vector of search direction and $\alpha_{q} 1$ is a scalar multiplier determining the amount of change in $\mathrm{X}$ for the current iteration. BFGS being a variable metric method, the search direction at iteration $\mathrm{q}$ is defined as

$$
S^{q}=-H \nabla F\left(X^{q}\right)
$$

where $\mathrm{H}$ is not Hessian matrix but approaches the inverse of the Hessian matrix for quadratic functions. At the initial design point, the $\mathrm{H}$ matrix is taken as the identity matrix $\mathrm{H}=\mathrm{I}$ and at the 
end of iteration q, a new matrix is obtained as

$$
H^{q+1}=H^{q}+\frac{1}{\gamma_{q}^{T} \sigma_{q}}\left(\beta_{q} \sigma_{q} \sigma_{q}^{T}-\sigma_{q} \gamma_{q}^{T} H_{q}-H_{q} \gamma_{q} \sigma_{q}^{T}\right)
$$

where

$$
\beta_{q}=1+\frac{\gamma_{q}^{T} H_{q} \gamma_{q}}{\gamma_{q}^{T} \sigma_{q}} \quad, \quad \sigma_{q}=X_{q+1}-X_{q} \quad, \quad \gamma_{q}=\nabla F\left(X^{q+1}\right)-\nabla F\left(X^{q}\right)
$$

Probably, the most useful form of iteration technique is the Golden Section Method. This needs many function evaluations, but is straightforward to program. The rate of convergence is known, and is very reliable for poorly conditioned problems. However, for improved global convergence, some modifications have been made to the original BFGS variable metric method which work well for the shape optimization problems.

Scaling also has a significant effect on performance of the optimization method because it helps to overcome the numerical difficulties which can arise during the process. Scaling the constraints and making them of the same order improves the optimization which is not then dominated by a single constraint. Scaling the objective function and make it be of order of unity in the region of interest is important, especially when the objective function is very small or very large and the termination criterion is based on absolute change of the objective function. Normalization of the search direction specifies the important design variables in the design region.

\section{STRESS ANALYSIS AND DESIGN SENSITIVITY ANALYSIS}

In the past ten years or so, the boundary element method has become increasingly successful in many branches of engineering science. It is replacing the FE technique for a wide range of applications in stress analysis, and is particularly well established in linear elastic problems. The method, instead of attempting to find an approximate solution for the governing differential equation throughout the solution domain, converts the equation into an integral form, often involving only integrals over the boundary of the domain. Consequently, the dimensionality of the problem is reduced effectively by one, so only the boundary has to be discretized in order to carry out the integration. In comparison with the finite element method, the mesh data preparation is greatly simplified and boundary elements are often more economical of 
computing time and storage. The boundary element approach offers continuous interior modelling within the solution domain of the problem, giving high resolution of stresses and displacements. But, the method has some drawbacks, and is less suitable for nonlinear problems. It is, however, particularly well suited for shape optimization problems.

When boundary element analysis is carried out, simultaneous equations of the form

$$
A U=B
$$

have to be solved, where $\mathrm{U}$ is the vector of unknown displacements or tractions, and $\mathrm{A}$ and $\mathrm{B}$ are the matrices which contain the integrals of the traction and displacement kernel functions, respectively. The solution of these equations gives the unknown displacements or tractions at the boundary.

The design sensitivity analysis part of the optimization algorithm is concerned with the calculation of the gradients of the objective and constraint functions with respect to the design variables. While it is possible to calculate these gradients numerically using difference schemes, the computational cost is relatively high, and the results of limited accuracy. This lack of accuracy can have an adverse effect on the rate of convergence of the optimization process. Here, the design sensitivity analysis is carried out by implicit differentiation of the structural response. This means differentiating the boundary integral equations analytically with respect to design variables $X_{q}$, which are the coordinates of some nodes of the movable part of the boundary,

$$
A \frac{\partial U}{\partial X_{k}}=\frac{\partial B}{\partial X_{k}}-U \frac{\partial A}{\partial X_{k}}
$$

This is a set of linear algebraic equations for the unknown gradients, $\frac{\partial U}{\partial X_{k}}$. The terms of the right hand side are known. The integrals of the traction and displacement kernels contained in A and $\mathrm{B}$ are dependent on the design variables, but only those elements which contain a particular design variable node will contribute nonzero values to the derivatives of A and B. In references [5-7] the derivatives of displacements and stresses with respect to design variables are calculated both by this direct analytical differentiation method and by the finite difference method. The former are shown to be both accurate and less time consuming. 


\section{BOUNDARY ELEMENT MESH GENERATION AND REGENERATION}

The input data required by the boundary element method for stress analysis are the geometry idealization, material properties and the loading and boundary conditions. The geometric representation of the structure by a suitable mesh is the most demanding of these: prepared manually, it is both time-consuming and liable to errors. In recent years, a wide variety of Computer Aided Design (CAD) packages have been developed for finite element data preparation and these can usually be adapted to create the much simpler boundary element surface meshes. Here the CAD software package IDEAS was used as a pre- and post-processor to generate boundary element meshes, create boundary conditions and tabulate physical and material properties. During the shape optimization process, the shape of the boundary changes and so does the boundary element mesh. An automated mesh generator and re-generator were therefore developed within the program which served as the interface between IDEAS and the boundary element code.

\section{SHAPE REPRESENTATION AND DESIGN VARIABLE SELECTION}

Shape representation uses design variables which are relevant to the boundary shape. In this work, the design variables are the coordinates of some points on the movable part of the boundary where shape is to be optimized. Hermitian cubic spline functions which are combinations of piecewise cubic curves, with continuous first and second derivatives at the ends of each curve, are used to represent boundary shape. This provides a flexible and compact method for defining boundary geometry using a small number of design variables. Additional geometrical optimization constraints that are generally necessary to avoid unrealistic designs are automatically taken into account [5-6].

\section{SHAPE OPTIMIZATION PROGRAM}

In order to apply the boundary element method for shape optimization to smoothing stress peaks for two-dimensional linear elastic problems, the general purpose computer program, STRESOPT in FORTRAN, was developed to study a number of problems [5]. This program uses an iterative technique and involves three major steps within each iteration:

i. An analysis of the stresses for a given design

ii. Sensitivity analysis corresponding to possible changes in this design 
iii. Improvements to the design, and generation of the boundary element mesh.

The program flowchart is shown in Fig. 1. The whole process is carried out automatically without user intervention. The results involve the geometry of the optimum shape, the shapes obtained during the iteration procedure and the corresponding stress distributions. All this information is stored in a universal file which IDEAS is able to read and then plot.

\section{NUMERICAL EXAMPLES}

Three examples are chosen to illustrate the use of the program. Firstly, a hole in an infinite thin plate in a biaxial stress field. The analytical solution for the tangential stress around the edge of an elliptical hole, with semi-major and semi-minor axes a and b, in an infinite plate due to a uniaxial stress field of intensity $\sigma$ acting at an angle $\alpha$ to the major axis of the ellipse (as in Fig. 2) is given by [12]

$$
\sigma_{\phi}=\sigma \frac{(1+K)^{2} \sin ^{2}(\phi+\alpha)-\sin ^{2} \phi-K^{2} \cos ^{2} \phi}{\sin ^{2} \phi+K^{2} \cos ^{2} \phi}
$$

In this equation $K=b / a$ and $\phi$ is the plane polar angular coordinate measured from the axis of the ellipse. The tangential stress due to a biaxial stress field of intensity $\sigma_{1}$ in the $\mathrm{x}$ direction (Fig. 2) parallel to the major axis of the ellipse and $\sigma_{2}$ in the y direction parallel to the minor axis can be obtained by summation. It is found that provided $\frac{\sigma_{2}}{\sigma_{1}}=K=\frac{b}{a}$ the tangential stress (and hence the von-Mises equivalent stress) is constant along the edge. This represents the optimum shape condition, when all the material at the edge of the hole is at the same maximum permissible stress.

The particular case chosen for analysis is that of $\frac{\sigma_{2}}{\sigma_{1}}=\frac{1}{2}$. The initial shape for the hole is taken as a circle. The problem is symmetric about both coordinate axis and only quarter of the plate need be considered. The boundary element model contains 36 quadratic elements, of which 12 are around the hole. The design variables are the radii of four points on the edge of the hole at equal angular intervals around it [5-6]. In Fig. 3, the optimum shape obtained numerically after 
25 iterations is shown together with the analytical solution. The same optimization problem has been solved using a finite element method, with a finite difference numerical method for the design sensitivity analysis [13]. Table 2 shows values of the ratio between the maximum stress at the edge of the hole, $\sigma_{\max }$, and the larger of the two biaxial stress components, $\sigma_{1}$. These values are for the present boundary element method and the finite element mehod for both the initial circular hole and the final optimized elliptical hole.

The second example is a double symmetric problem of four frame members meeting at a junction [13], as shown in Fig. 4. The shape of the fillets at the intersections of the frame edges is optimized for two forms of loading: when the frame is under biaxial tension and when it is being bent as shown. Because of symmetry, for the biaxial loading only one quarter of the frame need be modelled, while for the bending case half of the frame was considered. Totals of 36 and 78 boundary elements were used for biaxial and bending cases, respectively, in each case 12 elements being used around each fillet.

The radii of 5 points at equal angular intervals around each fillet were considered as design variables. Figure 5 shows the optimum solution for both loading cases, together with the initially assumed fillet shape. The same problem has been solved using finite elements [13]. Table 2 shows for comparison the values of stress concentration factor for each method under the two types of loading for both the initial and optimized designs.

The third example concerns the selection of the best shape of fillet in a stepped tension bar so that no yielding occurs [14]. The geometrical details of the fillet are shown in Fig. 6: symmetry of the problem means that only the upper half of the bar need be considered.

Figure 7 shows the initial and optimum designs, the latter being oriented after 10 iterations. There is a $30 \%$ reduction in the objective function and the stresses around the profile become more uniform while the maximum stresses before and after minimization are similar. In this case the design variables are the radii of 7 points at equal angular intervals around the profile BC (Fig. 6), while the edge $\mathrm{CD}$ remains horizontal and the profile meets $\mathrm{AB}$ at right angles. 


\section{CONCLUSION}

A computational approach to structural shape optimization has been presented. This uses the boundary element method for stress analysis and design sensitivity analysis together with facilities for numerical optimization, shape representation, and boundary element mesh regeneration. The numerical optimization method used is the BFGS variable metric method. The method is very suitable because the objective function, the square of the deviation of the equivalent stresses around the movable part of the boundary from a desired stress, is highly nonlinear while the side constraints upon the design variables are linear.

The program STRESOPT which implements this approach has been applied to the shape optimization of fillets and holes of inappropriate shape which are known contributors to premature failure of mechanical components. The boundary element method, being a boundary-oriented technique, is very appropriate for this purpose. It can overcome a number of the difficulties associated with its main rival, the finite element method. First, finite element mesh regeneration during the optimization process is costly and time-consuming while with boundary elements only the boundary of the structure need be modelled, and the design variables and shape changes are restricted to the boundary. Secondly, the computation of the derivatives in the sensitivity analysis, an essential step in the optimization process, in finite element methods is usually carried out by a finite difference scheme. Using boundary elements, these derivatives can be found by differentiating the integral equations analytically, which is less costly and more accurate.

\section{REFERENCES}

1. Vanderplaats, G. N., "Numerical Optimization Techniques for Engineering Design with Applications", McGraw-Hill, London (1984)

2. Fox, R. L., "Optimization Methods for Engineering Design" ,Addison-Wesely, New York (1971)

3. Rao, S. S., "Optimization Theory and Applications", Wiley, India, (1978)

4. Walsh, G. R., "Methods of Optimization", Wiley, Chichester (1975) 
5. Tafreshi, A., "Design Optimization Using Boundary Element Method", PhD Thesis, Imperial College, University of London (1990)

6. Tafreshi, A. and Fenner, R.T., "Design Optimization Using the Boundary Element Method", J. Strain Analysis, 26, 231-241 (1991)

7. Tafreshi, A. and Fenner, R.T., "Design Sensitivity Analysis Using the Boundary Element Method, J. Strain Analysis, 28, 283-291 (1993)

8. IDEAS (Integrated Design Engineering Analysis Software), "Finite Element Modelling User's Manual", Structural Dynamics Research Corporation, USA (1990)

9. Broydon, C. G., "The Convergence of a Class of Double Rank Minimization Algorithms", Parts I and II, J. Inst. Math. Appl., 6, 76-90, 222-231 (1970)

10. Fletcher, R., "A New Approach to Variable Metric Algorithms", Computer J., 13, 317 322 (1970)

11. Goldfarb, D., "A Family of Variable Metric Methods Derived by variational means", Math. Comp., 24, 23-36 (1970)

12. Muskhelishivili, N. I., "Some Basic Problems of the Mathematical Theory of Elasticity", Izd. Akad. Nauk, Moscow (1935)

13. Kristensen, E. S. and Fleming Madsen, N., "On the Optimum Shape in Fillets in Plates Subjected to multiple in plane loading cases", Int. J. Num. Meths in Engng, 10, 1007-1019 (1976)

14. Haug, E. J., Choi, K. K. and Komkov, V., "Design Sensitivity Analysis of Structural Systems", Academic Press, Inc., New York (1986) 
Tafreshi, A. \& Fenner, R. T. 3 Sep 1995 In : Computers and Structures. 56, 5, p. 713-720 8 p.

Table 1 Analytical and numerical values of $\frac{\sigma_{\max }}{\sigma_{1}}$ for the elliptical hole problem

\begin{tabular}{|c|c|c|c|}
\hline & BEM [5] & FEM [13] & Analytical \\
\hline Circular hole & 2.51 & 2.27 & 2.50 \\
\hline Optimum shape & 1.59 & 1.43 & 1.50 \\
\hline
\end{tabular}

Table 2 Stress concentration factors for the optimum web frame under biaxial and bending loading

\begin{tabular}{|c|c|c|c|c|}
\hline & \multicolumn{2}{|c|}{ BEM } & \multicolumn{2}{c|}{ FEM [13] } \\
\hline & Biaxial & Bending & Biaxial & Bending \\
\hline Initial design & 1.70 & 1.98 & 1.51 & 1.93 \\
\hline Optimum design & 1.32 & 1.63 & 1.31 & 1.64 \\
\hline
\end{tabular}




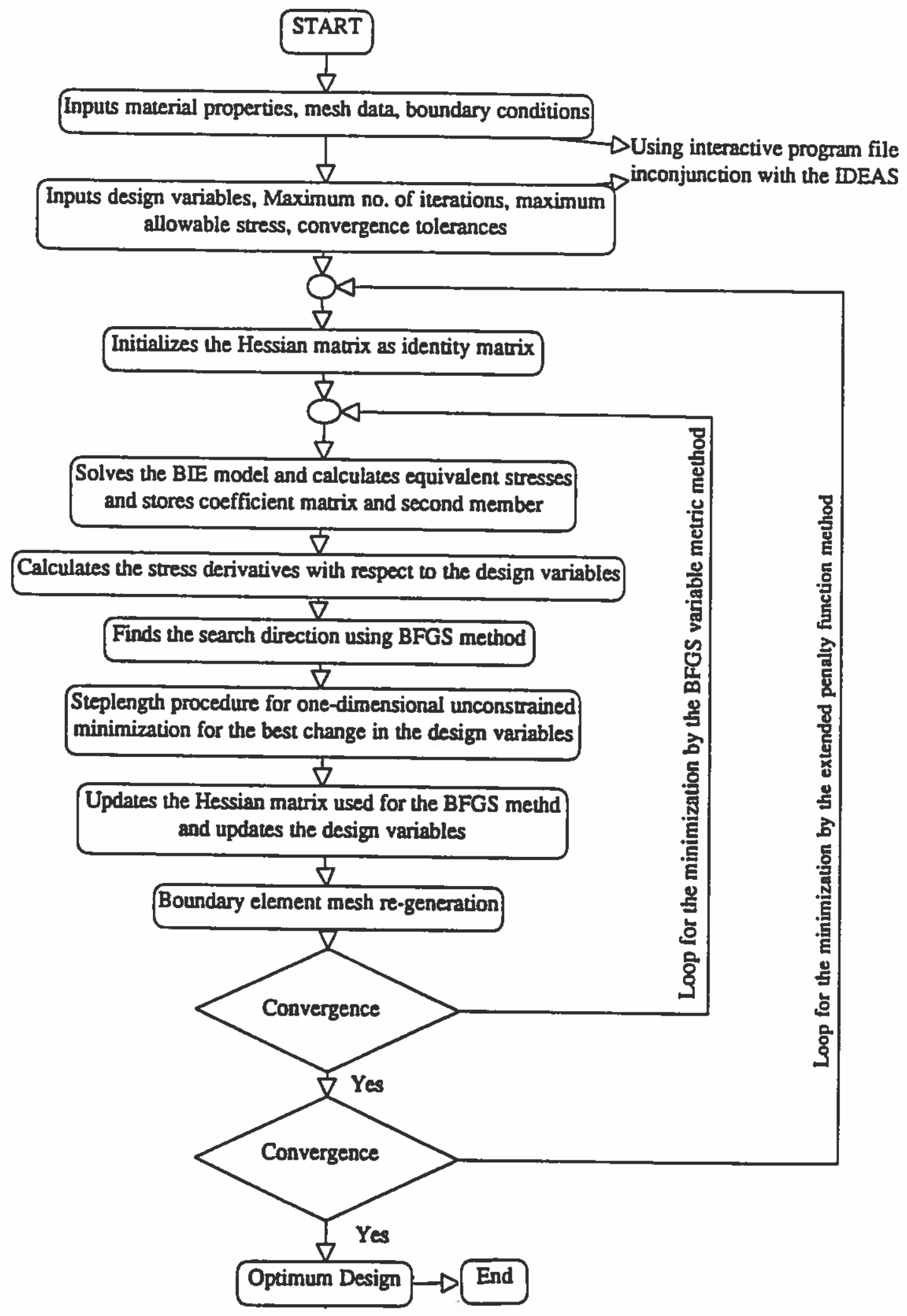

Fig. 1 Flow chart for the peak stress minimization 


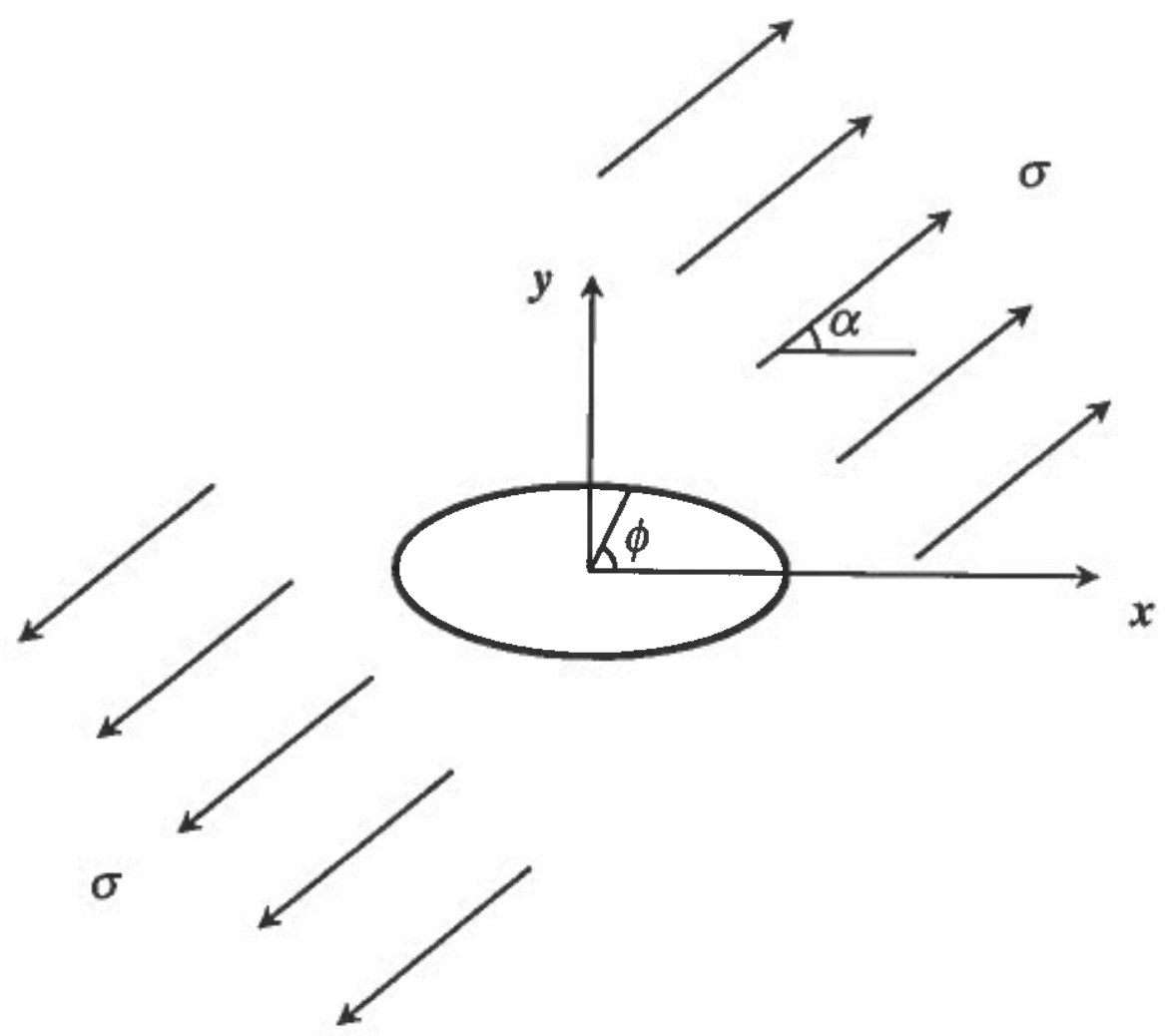

Fig. 2 Elliptical hole in an infinite plate under a biaxial stress field 
Tafreshi, A. \& Fenner, R. T. 3 Sep 1995 In : Computers and Structures. 56, 5, p. 713-720 8 p.

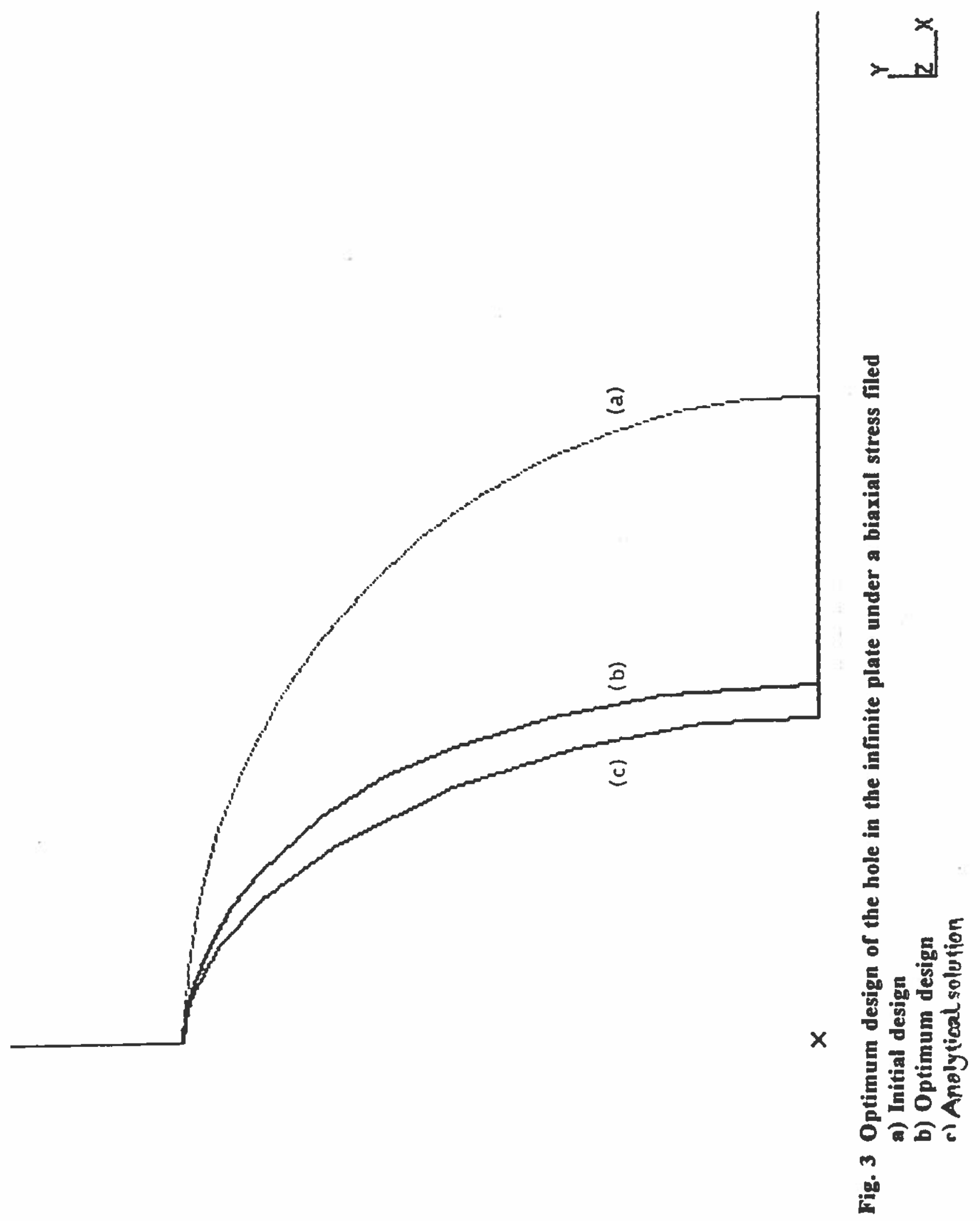


Tafreshi, A. \& Fenner, R. T. 3 Sep 1995 In : Computers and Structures. 56, 5, p. 713-720 8 p.

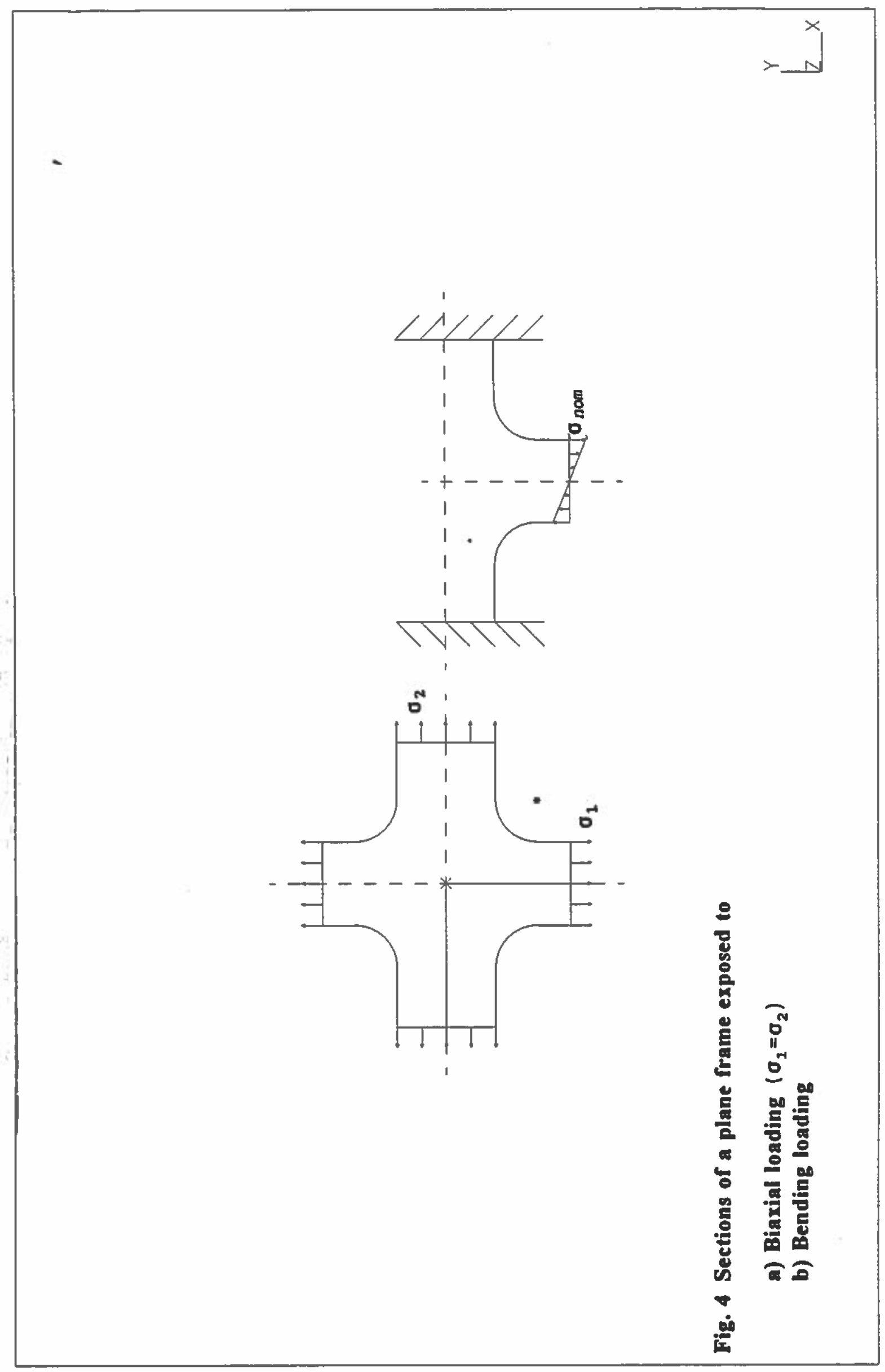


Tafreshi, A. \& Fenner, R. T. 3 Sep 1995 In : Computers and Structures. 56, 5, p. 713-720 8 p.

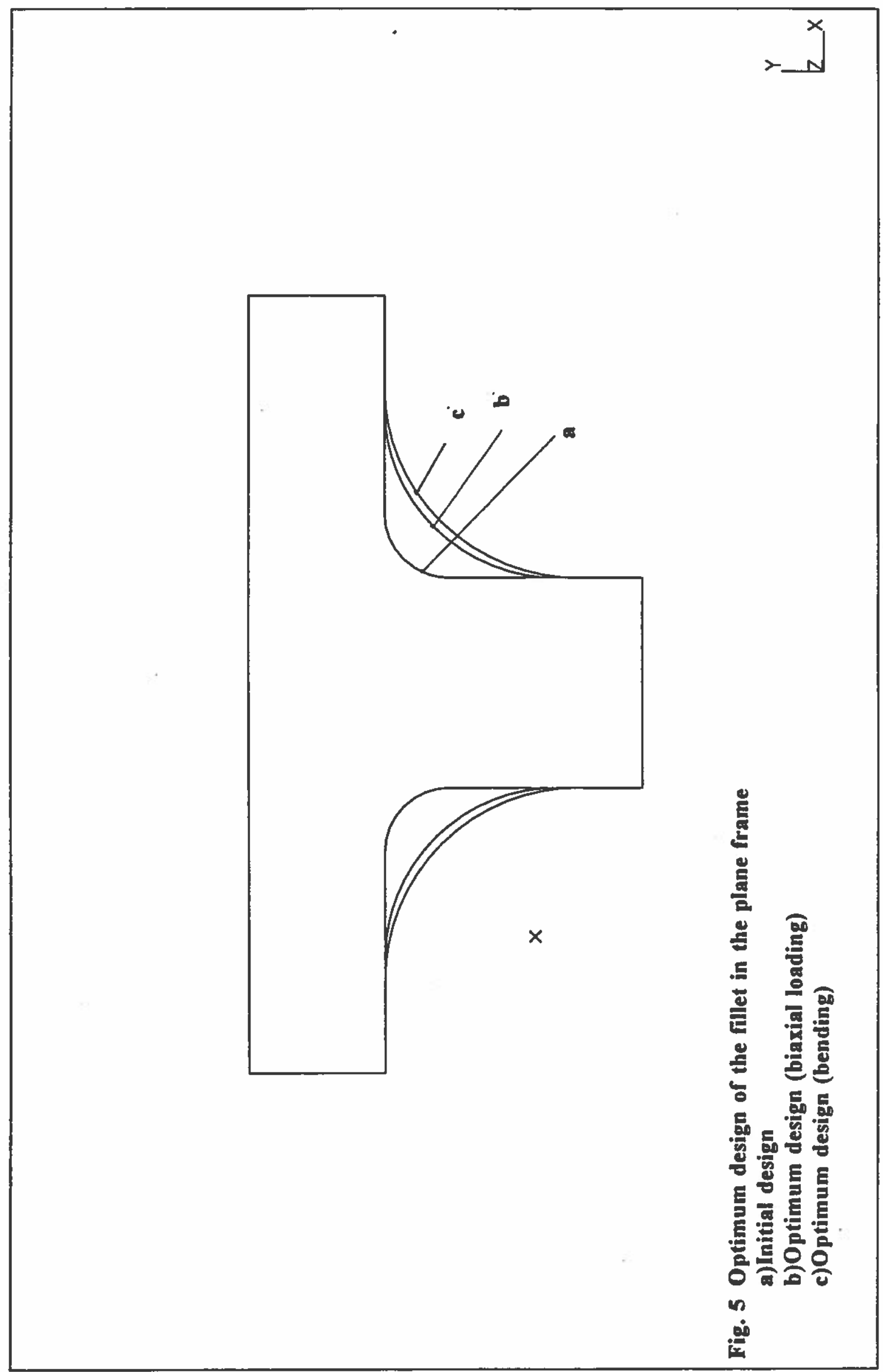


Tafreshi, A. \& Fenner, R. T. 3 Sep 1995 In : Computers and Structures. 56, 5, p. 71̣-720 8 p.

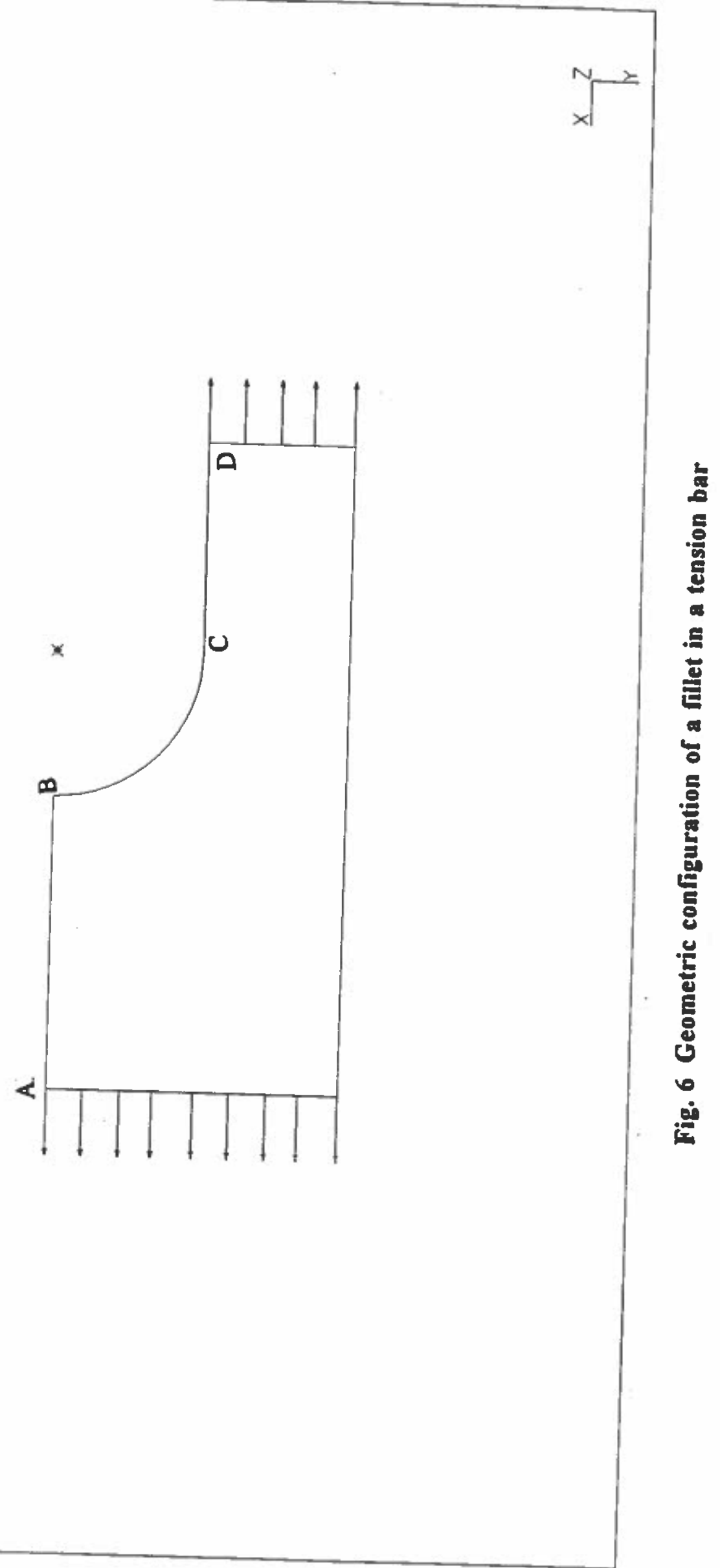


Tafreshi, A. \& Fenner, R. T. 3 Sep 1995 In : Computers and Structures. 56, 5, p. 713-720 8 p.
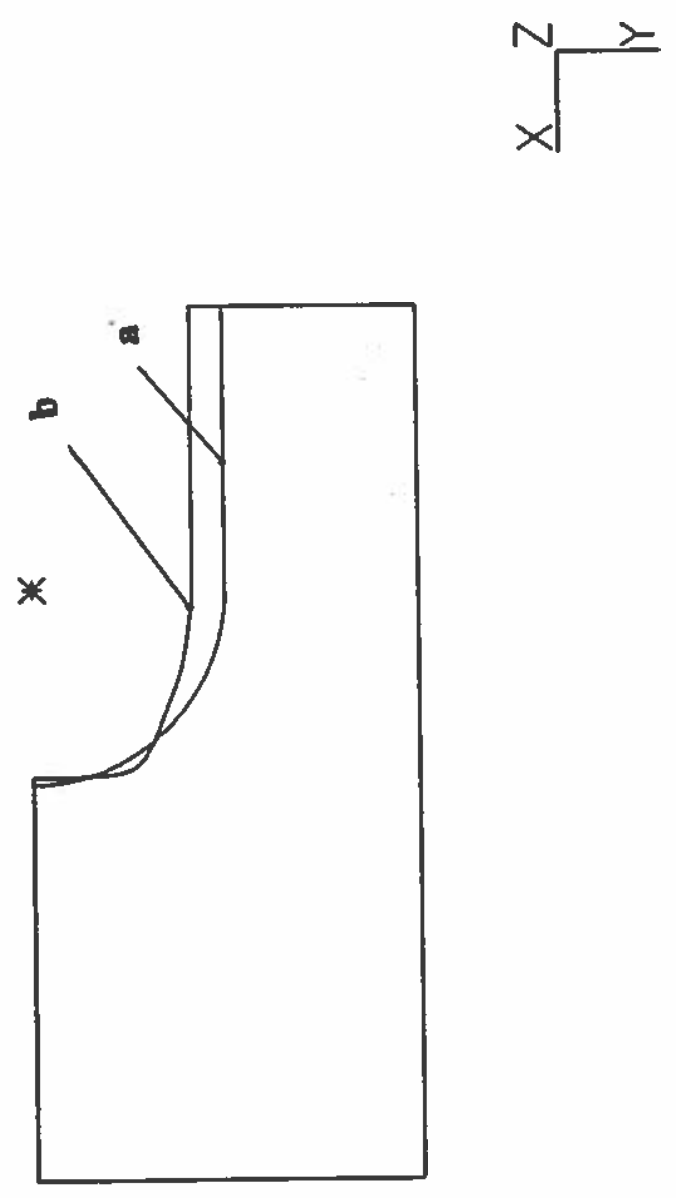

등 